J. Lake Sci.(湖泊科学), 2016, 28(2): 455-462

DOI 10. 18307/2016. 0226

(C) 2016 by Journal of Lake Sciences

\title{
乌梁素海冰封期湖泊冰盖组构特征对污染物分布的影响”
}

\author{
杨 芳, 李畅游**史小红, 赵胜男, 郝亚洲 \\ ( 内蒙古农业大学水利与土木建筑工程学院,呼和浩特 010018)
}

\begin{abstract}
摘 要: 为探究富营养化浅水湖泊季节性冰盖污染物分布规律,于 2013-2014 年冰封期,钻取乌梁素海湖泊冰盖冰芯试 样, 观测冰厚并对冰芯晶体结构、气泡含量、污染物浓度 (总氮、总磷和 $\mathrm{COD}_{\mathrm{Cr}}$ ) 进行分析. 结果表明: 冰盖可分为 4 层, 中 间 2 层冰晶体粒径较大且气泡含量较少, 为冰盖热力生长区. 冰盖以柱状晶体居多, 粒径随深度增加而增加, 气泡含量随 冰盖密度增加而减少. 冰盖结构特征与污染物分布具有相关关系, 冰芯密度及气泡分布与总氮、总磷和 $\mathrm{COD}_{\mathrm{Cr}}$ 相关关系分 别为 $0.8965 、 0.8718 、 0.8184$, 并建立多元回归模型揭示冰封期湖泊水质特征, 为季节性湖泊冰盖研究及冰封期湖泊水资源 规划和管理提供理论依据.
\end{abstract}

关键词: 污染物分布; 冰封期;冰盖结构;冰体杂质;乌梁素海

\section{Impact of seasonal ice structure characteristics on ice cover impurity distributions in Lake Ulansuhai}

YANG Fang, LI Changyou ** , SHI Xiaohong, ZHAO Shengnan \& HAO Yazhou

(Inner Mongolia Agricultural University, College of Water Conservancy and Civil Engineering, Hohhot 010018, P.R.China)

\begin{abstract}
Investigation and analysis of ice core on Lake Ulansuhai from ice season of 2013 to 2014, observational parameter included ice thickness, examination of ice crystal type and bubble structure contributing to the ice cover, measurements of total nitrogen $(\mathrm{TN})$, total phosphorus ( TP $)$ and chemical oxygen demand $\left(\mathrm{COD}_{\mathrm{Cr}}\right)$. The preliminary analyses were as follows: the ice cores were divided into four layers. The two middle layers were the mainly thermal ice growth areas. The ice cover was consisted of columnar-crystal ice for the most part, and the crystal size increased with the ice-cover depth and the gas content decreased with the increase of density. The content of contaminates $\left(\mathrm{TN}, \mathrm{TP}, \mathrm{COD}_{\mathrm{Cr}}\right)$ were significantly correlated with density and gas content $\left(r_{\mathrm{TN}}=\right.$ $0.8965, r_{\mathrm{TP}}=0.8718$ and $\left.r_{\mathrm{COD}}=0.8184\right)$, and we respectively established the multiple regression equation to reveal the lake water quality characteristics in frozen period. It provides theory basis for seasonal ice-cover study and water resources planning and management in ice season.
\end{abstract}

Keywords: Nutrient distributions; ice season; ice crystal structure; ice impurities; Lake Ulansuhai

中国湖泊按照自然环境、区域分异和水资源特征,分为东北平原与山地区、蒙新高原区、青藏高原区、东 部平原区和云贵高原区五大湖区 ${ }^{[1]}$. 蒙新高原区的乌梁素海地处高纬度地区, 湖泊的冬季冰封时间长, 冰盖 的生长将改变湖泊热交换过程、污染物分布特征及冰下底栖温度环境 ${ }^{[2]}$. 在污染物浓度较高的浅水湖泊中 冰盖总体积约占全湖总体积的 $1 / 2 \sim 2 / 3$, 季节性冰盖既是污染物载体, 又是冬季冰下液态水成为高浓缩状 态的驱动因素之一 ${ }^{[3-4]}$, 因此研究湖冰环境下污染物的迁移转化过程与湖泊冰芯结构特征, 对于研究湖泊生 长机制及深人研究寒区湖泊污染机理不容忽视.

然而在整个冰封期中进行湖泊冰盖生长过程的连续观测非常困难, 姜慧琴等 ${ }^{[5-6]}$ 采用室内实验模拟不 同 $\mathrm{pH}$ 值及温度条件下, 乌梁素海水体冻、融过程中营养盐及重金属的释放规律; 张岩等 ${ }^{[7]}$ 通过室外人工池 模拟研究乌梁素海冰封期实际气温条件下, 冰体生长特征及污染物迁移规律. 然而模拟实验忽略了众多影

* 国家自然科学基金重点项目 (51339002) 和国家自然科学基金项目 (51269017,51169017) 联合资助.2016-01-05 收稿;2016 -01 - 11 收修改稿. 杨芳( 1986 ),女,博士研究生;E-mail:ffff.yyyy@ sina.com.

** 通信作者;E-mail:nndlichangyou@163.com. 
响因素, 结果势必与自然状态有一定差距. Leppäranta 认为湖泊冰芯样品组构分析可以获取存储在冰盖各层 及冰晶体中的冰体生长过程的信息 ${ }^{[2]}$; Gow 和 Govoni 通过观测温带季节性冰盖的物理和结构特征用于研究 冰盖垂向生长规律 ${ }^{[8]}$. Li 等通过人工冰和自然冰对比发现, 在淡水冰盖纵向剖面中, 冰晶体类型、晶体尺寸、 气泡形状、气泡大小及百分含量随着冰体密度自上而下变化着 ${ }^{[9-10]}$. Shi 等在南极海冰研究中, 认为冰盖晶体 组构与热能散失的关系对于冰体物理特征及其盐度分布有重要意义 ${ }^{[11]}$. 因此,本文利用 2013-2014 年冰封 期在内蒙古乌梁素海取得的冰芯试样, 首次从冰晶结构、冰密度及污染物分布等方面, 分析乌梁素海湖泊冰 盖特征及污染物分布规律, 研究冰封期浅水富营养化湖泊冰盖生长机制.

\section{1 材料与方法}

\section{1 研究区概况}

乌梁素海 $\left(40^{\circ} 36^{\prime} \sim 41^{\circ} 03^{\prime} \mathrm{N}, 108^{\circ} 43^{\prime} \sim 108^{\circ} 57^{\prime} \mathrm{E}\right.$ ) 位于内蒙古自治区西部, 河套平原东端, 乌拉特前旗境 内, 水深 $1.4 \sim 3.1 \mathrm{~m}$, 水面面积为 $283 \mathrm{~km}^{2}$, 芦苇区占湖面的 $45.8 \% \sim 51.5 \%$, 是全球范围内同纬度荒漠半荒 漠干旱地区中最大的湿地草型湖泊, 也是中国第八大淡水湖, 已列入国际重要湿地名录. 主要承纳来自河套 灌区农田退水及周边村镇的工业废水与生活污水, 经红圪卜扬水站汇人湖区, 最终由西山咀汇人黄河, 其中 农业退水携带有大量营养盐一同汇人, 加速了乌梁素海的富营养化. 湖泊所在地区四季更替明显, 多年平均 气温为 $7.3^{\circ} \mathrm{C}$,一般从每年 11 月初进人冰封期, 到次年 3 月末至 4 月初开始融化, 约有 $4 \sim 5$ 个月处于冰层覆 盖阶段. 多年冰盖平均厚度为 0.63 m. 2013 年 10 月 24 日薄冰初现到 2014 年 4 月 15 日冰盖开始消融期间, 最低气温为 $-20.1^{\circ} \mathrm{C}$, 整个冰封期连续气温零度以下共持续 $103 \mathrm{~d}$.

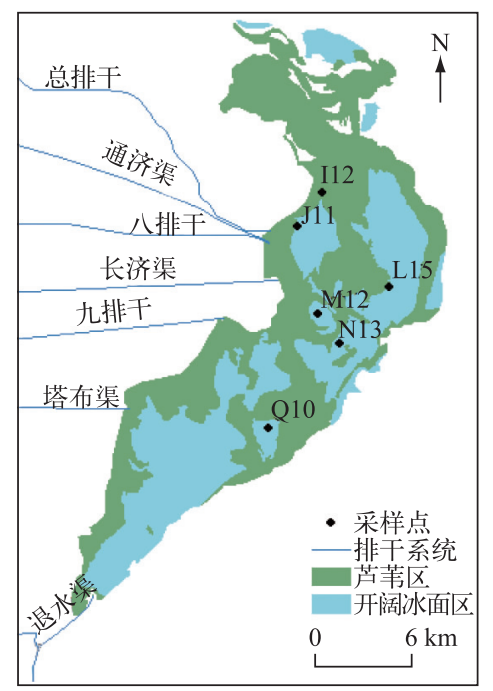

图 1 乌梁素海采样点示意

Fig.1 Distribution of sampling sites of Lake Ulansuhai

\section{2 采样方法}

出于安全及样品完整性考虑, 根据多年气象资料, 一般在每 年的 12 月底到 1 月中旬, 即乌梁素海进人完全冰封阶段, 进行冬 季样本采集和现场监测工作. 在乌梁素海主要湖区布设现场监测 点(图 1), 于 2014 年 1 月 16 日, 利用 GPS 定位系统在湖上确定 监测点位置进行取样.

在每个测点标记正北方向, 拍照记录, 每间隔 $2 \mathrm{~m}$ 钻取 3 根 完整冰芯试样 $(\mathrm{V} 、 \mathrm{H} 、 \mathrm{C})$ 并标记正北, $\mathrm{H}$ 冰芯试样进行水平晶体切 片和内部气泡分布分析; V 冰芯试样用作垂向晶体结构切片及宏 观气泡形态观测; C 冰芯试样作为冰密度测量及备份. 在冷库 $-15^{\circ} \mathrm{C}$ 的条件下, 将冰芯以 5 7 cm 一层进行分割, 分别制备水平 及坚直方向的切片. 图 2a 为 I12 纵向切片, 将所需断面贴在略高 于 $0^{\circ} \mathrm{C}$ 的玻璃片上, 标记该样品信息, 用刨刀将冰样磨薄至 $1 \sim 2$ $\mathrm{mm}$ ( 取决于单层气泡的厚度). 在黑色背景下观测气泡分布、正交 偏光镜费氏台下观测冰晶结构 ${ }^{[12]}$. 第 3 根冰芯试样分层后, 采用 质量体积法进行密度测量, 融化后进行污染物浓度检测. 总氮 (TN) 浓度采用过硫酸钾氧化紫外线分光光度法测定, 总磷 (TP) 浓度采用钿锑抗分光光度法测定, 化学需氧量 $\left(\mathrm{COD}_{\mathrm{Cr}}\right)$ 浓度采用 密封消解法进行测定 ${ }^{[13]}$.

受湖泊中不同水深、流速和水生植物疏密程度的影响, 采样点的冰芯结构存在差异, 但总体上, 冰盖的 结构可分为表层 (初冰层)、中层、近底层和底层. I12 点的水深和流速为全湖平均水平, 水生植物较少, 冰芯 较完整, 在本文中可以代表整个湖泊冰盖的基本特征, 因此以 I12 点为例描述乌梁素海冰盖内部结构特征.

\section{2 结果}

\section{1 冰盖中污染物垂向分布特征}

对乌梁素海 3 组冰芯试样的污染物进行分析, 由于各采样点的冰盖厚度不同, 按每 $5 \mathrm{~cm}$ 为一层进行划 


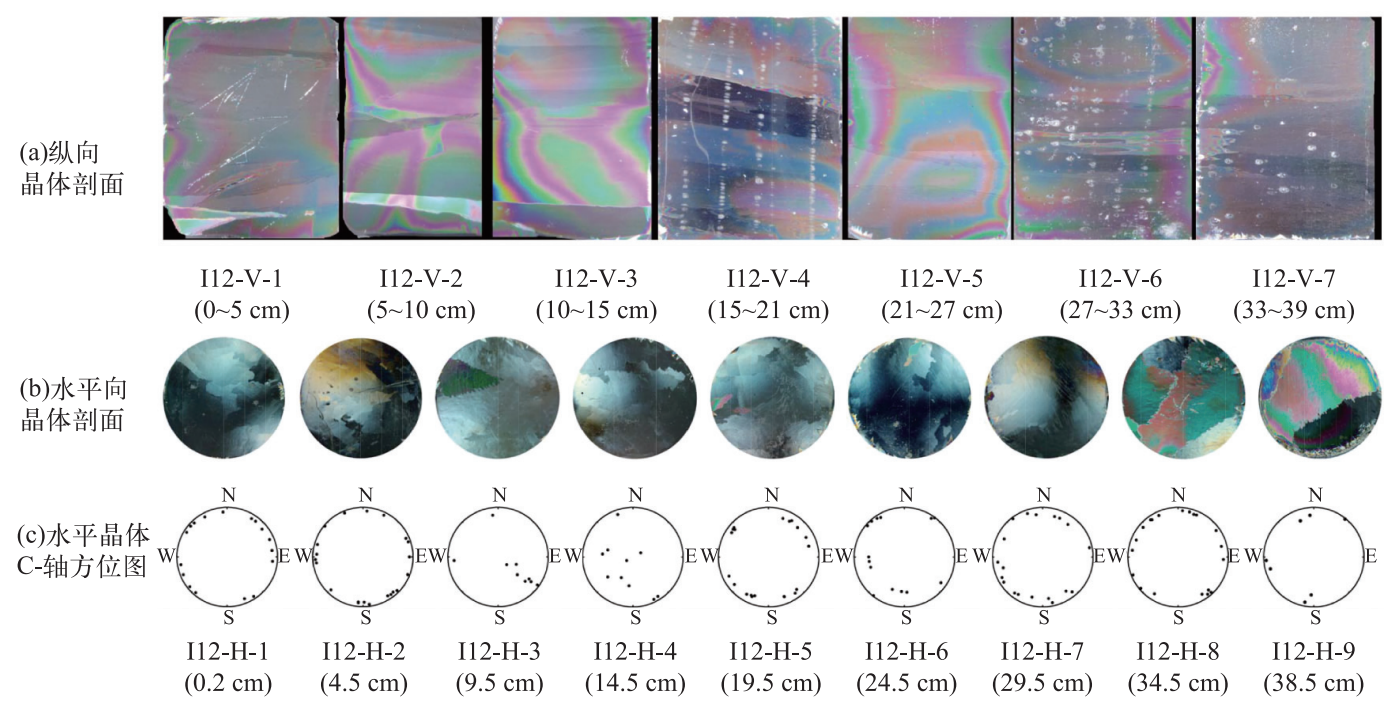

图 $2 \mathrm{I} 12$ 冰芯纵向及水平向晶体剖面和水平晶体 $\mathrm{C}$-轴方位分布

Fig. 2 Vertical and horizontal crystal profiles and crystal C-section of I12 ice core

分的层数就不同, 为了能够更清晰地反映冰盖中污染物的垂向分布特征, 以每个冰芯试样厚度为其单位厚 度,将不同深度的污染物浓度折算成冰盖厚度百分比对应的污染物浓度. 图 3 为乌梁素海冰芯试样中 TN、 $\mathrm{TP}$ 和 $\mathrm{COD}_{\mathrm{Cr}}$ 浓度垂向分布. 不同采样点的各污染物浓度垂向变化有所差异, 总体上随着深度的增加呈现出 先减小后增大的趋势. 表层 $(0 \sim 2.5 \%)$ 和底层 $(85 \% \sim 100 \%)$ 的污染物浓度较大, 中层 $(2.5 \% \sim 50 \%)$ 及近底 层 $(50 \% \sim 85 \%)$ 冰体中的污染物浓度较低.

\section{2 冰盖内部组构特征}

2.2.1 冰晶体类型和晶体尺寸分析 $\mathrm{I} 12$ 点的冰芯试样 $\mathrm{H}$ 全长 $39 \mathrm{~cm}$, 按每 $5 \mathrm{~cm}$ 一层取水平剖面, 其中 I12-1 为 $0.2 \mathrm{~cm}$ 处, 为该点冰盖首层剖面, 共制作 9 个水平切片. $\mathrm{I} 12-\mathrm{H}$ 冰芯试样水平剖面晶体粒径分布见表 1. 表 层 (I12-1) 即初冰层, 晶粒较细碎, 为多边形, 晶体平均粒径为 $9.60 \mathrm{~mm}$, 最大粒径为 $14.79 \mathrm{~mm}$, 都为整个冰 芯最小值; I12-2、I12-3、I12-4 代表冰芯中层, 结合 I12 点的冰芯试样 $V$ 的纵向剖面 (图 2a) , 随着冰盖厚度的 增加, 晶体结构以柱状冰为主, 冰晶体粒径有所增加, 平均粒径为 $20.25 \mathrm{~mm}$, 冰芯试样最大粒径为 29.94 $\mathrm{mm}$; I12-5、I12-6、I12-7、I12-8 为冰芯试样近底层, 冰晶体粒径呈先增大后减小的变化趋势, 平均粒径为 16.52 $\mathrm{mm}$, 冰芯试样最大粒径为 $24.05 \mathrm{~mm}$; I12-9 为冰芯试样底层, 冰晶体颗粒尺寸突增且个数减少, 平均粒径为 $29.08 \mathrm{~mm}$, 最大粒径为 $34.02 \mathrm{~mm}$.

表 1 I12-H 冰芯试样水平剖面晶体粒径分布

Tab.1 Crystal size distribution in horizontal section of point I12

\begin{tabular}{lccccccccc}
\hline & $\mathrm{I} 12-1$ & $\mathrm{I} 12-2$ & $\mathrm{I} 12-3$ & $\mathrm{I} 12-4$ & $\mathrm{I} 12-5$ & $\mathrm{I} 12-6$ & $\mathrm{I} 12-7$ & $\mathrm{I} 12-8$ & I12-9 \\
\hline 水平切片位置/cm & 0.2 & 6 & 10 & 15 & 20 & 25 & 30 & 35 & 39 \\
平均粒径 $/ \mathrm{mm}$ & 9.60 & 16.46 & 23.58 & 20.72 & 15.29 & 20.63 & 14.99 & 15.15 & 29.08 \\
最大粒径 $/ \mathrm{mm}$ & 14.79 & 19.60 & 29.94 & 27.14 & 20.12 & 24.05 & 20.65 & 19.19 & 34.02 \\
\hline
\end{tabular}

晶体 C-轴特征可以体现冰盖的光学特征和物理性质. 将 I12-H 的水平切片置于费氏旋转台上, 读取方位 角和倾角,投影后绘制在 Schmidt 等面积正交网格上,得到 C-轴的组构图 ${ }^{[14]}$. 如图 2c 所示, I12-1-H 和 I12-2-H 的晶体 C-轴大致为环形分布, 呈现随机分布规律, 为各向同性材料, 在 I12-3-H I12-4-H 平面内稍有优势取 向, 属于平面内定向分布, 方向为 NNW-SSE, 因此冰盖的中层为各向异性材料, 上层、近底层及底层为各向同 性材料. 


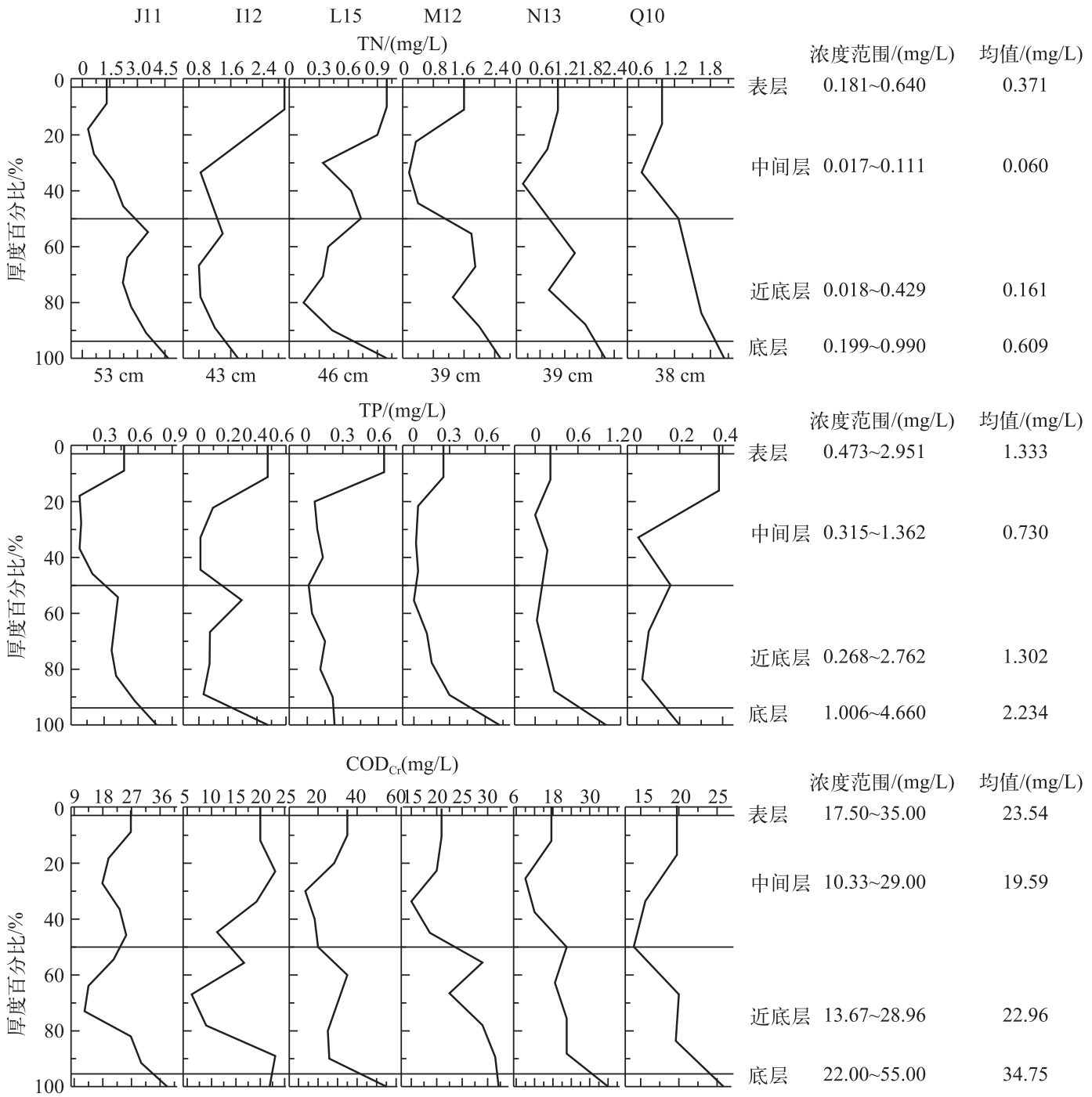

图 3 冰芯内总氮、总磷、COD ${ }_{\mathrm{Cr}}$ 浓度垂向分布

Fig.3 Distribution of total nitrogen, total phosphorus and $\mathrm{COD}_{\mathrm{Cr}}$ concentrations along vertical direction in ice cores

2.2.2 冰芯气泡特征 冰芯气泡含量不仅可以影响冰盖的导热系数, 而且从一定程度上改变了冰下水体与冰 上大气的导热通道和形式 ${ }^{[15]}$. 同时由于空气的折射率不同于纯水,导致光的折射、反射、散射途径改变,使 得进人冰下水体的日照强度和辐射总量减弱, 影响水生生物的生长 ${ }^{[16]}$, 所以气泡含量是冰盖重要的物理特 征. 对冰芯试样纵向切片在正常光照射下进行拍照,如图 3, 借鉴李志军等 ${ }^{[12]}$ 的冰晶体图像分析方法, 得到 气泡数据, I12 冰芯试样纵向剖面气泡特征见表 2, 可将 $0 \sim 5 \mathrm{~cm}$ 层视为表层, 该层气泡稀疏且等效直径变化 范围较大; $5 ~ 20 \mathrm{~cm}$ 位于冰芯试样中层, 气泡较少且较细小; $20 \sim 32 \mathrm{~cm}$ 位于冰芯试样近底层, 气泡含量明显 增加, 垂向层状分布, 其气泡等效直径较大; $32 \sim 39 \mathrm{~cm}$ 层为冰芯试样底层, 气泡较大且出现层状分布. 整个 冰芯气泡平均含量为 $0.158 \% \sim 8.050 \%$, 平均等效直径为 $0.324 \mathrm{~mm}$, 最大可达 $9.961 \mathrm{~mm}$.

2.2.3 密度特征 密度也是反映冰盖结构特征的重要参数, 天然淡水冰盖是纯冰晶、气泡和杂质包裹体的混 合物, 故其密度随其内部各组分含量变化而变化 ${ }^{[17]}$. 全湖冰盖密度在垂向上变化趋势较一致. 
表 2 I12 冰芯试样纵向剖面气泡特征

Tab.2 Gas bubble characteristic in vertical section of I12 ice core

\begin{tabular}{|c|c|c|c|c|}
\hline \multirow{2}{*}{ 深度/cm } & \multirow{2}{*}{ 气泡含量/\% } & \multicolumn{2}{|c|}{ 气泡等效直径/mm } & \multirow{2}{*}{ 气泡形状 } \\
\hline & & 平均直径 & 最大直径 & \\
\hline $0 \sim 5$ & 0.579 & 0.215 & 1.442 & 圆形和近圆形 \\
\hline $5 \sim 10$ & 0.362 & 0.200 & 0.658 & 圆形和近圆形 \\
\hline $10 \sim 15$ & 0.158 & 0.190 & 0.551 & 圆形和近圆形 \\
\hline $15 \sim 20$ & 0.579 & 0.680 & 9.961 & 层状分布,近圆形 \\
\hline $20 \sim 25$ & 2.960 & 0.303 & 1.404 & 层状分布,近圆形 \\
\hline $25 \sim 32$ & 8.050 & 0.437 & 2.079 & 层状分布,近圆形, 部分柱状和针状 \\
\hline $32 \sim 39$ & 3.951 & 0.243 & 2.141 & 层状分布, 近圆形, 少许柱状和针状 \\
\hline
\end{tabular}

由于各采样点的冰厚度不一, 为方便横向比较, 以每个冰芯厚度为其单位厚度, 将不同深度的密度折算 成冰厚百分比对应的密度 (图 4). 表层冰芯密度较小, 变化范围为 $0.79 \sim 0.93 \mathrm{~g} / \mathrm{cm}^{3}$, 平均值为 $0.86 \mathrm{~g} / \mathrm{cm}^{3}$; 中 层密度稍有增大, 在 $0.88 \sim 0.96 \mathrm{~g} / \mathrm{cm}^{3}$ 之间变动, 平均值为 $0.91 \mathrm{~g} / \mathrm{cm}^{3}$; 近底层密度较均匀, 变化范围为 $0.87 \sim$ $0.94 \mathrm{~g} / \mathrm{cm}^{3}$, 平均值为 $0.90 \mathrm{~g} / \mathrm{cm}^{3}$; 底层部密度较大, 在 $0.86 \sim 0.96 \mathrm{~g} / \mathrm{cm}^{3}$ 之间变动, 平均值为 $0.91 \mathrm{~g} / \mathrm{cm}^{3}$.

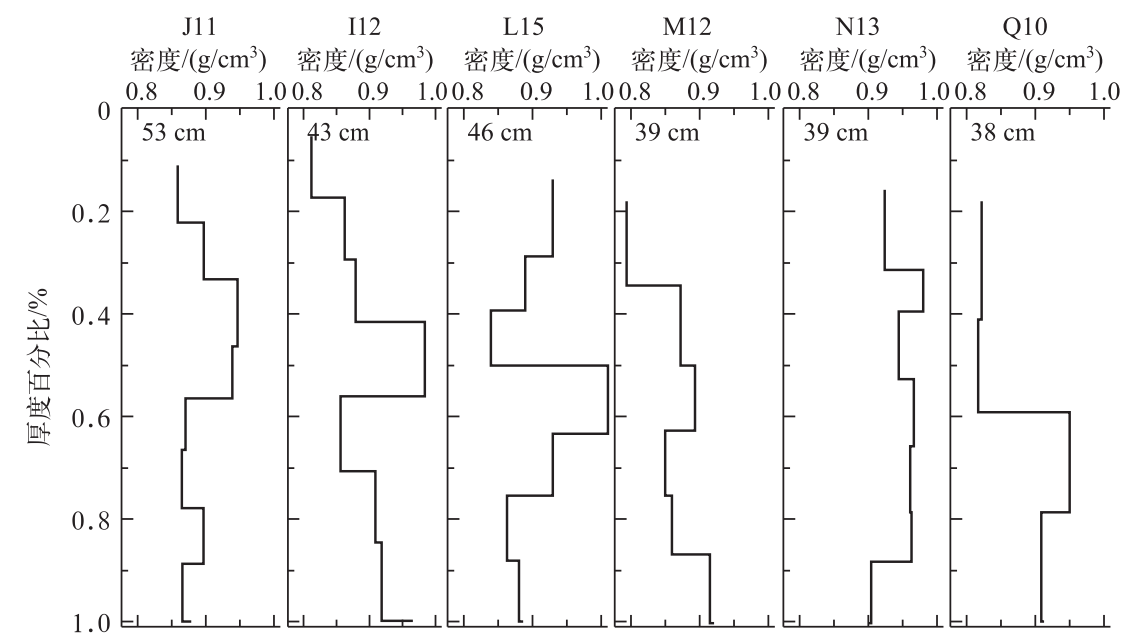

图 4 冰盖垂向密度变化

Fig.4 Ice density changes along vertical direction in ice core

\section{3 讨论}

每年 10 月末至 11 月中旬乌梁素海迎来第一次大幅度降温, 夜间湖水表层结冰, 若遇次日晴暖, 白天即 可融化. 直至 12 月初, 日间温度也降至零度以下, 由于日间的阳光照射和湖水扰动, 部分初冰发生融化或碎 裂, 但已处于过冷状态, 若遇夜间温度骤降, 初次冰盖就此形成, 全湖平均为 $1.3 \mathrm{~cm}$. 此时冰晶体发育不完

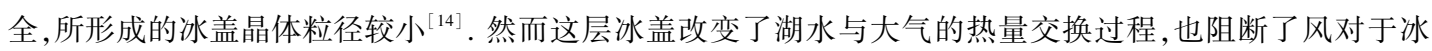
下水体的扰动, 随着低温的持续, 冰晶体在较为稳定的环境中开始生长, 晶粒尺寸显著增加; 由于受到相邻 晶体水平方向上的挤压限制, 晶体选择向下延伸 ${ }^{[18]}$, 此时形成的冰盖多为柱状冰, 主要以热力生长为主, 为

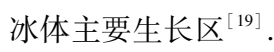

冰体冻结过程中, 初冰往往在相对较短的时间内完成, 湖水由液相转化为固相时, 溶解在液相中的气体 来不及逃逸, 形成气泡镶嵌在冰体内 ${ }^{[20]}$, 由于形成环境与降水, 湖面风浪的扰动, 湖水温度的不稳定等因素 的影响, 表层的气体细小繁多, 分布杂乱; 中层冰体由于已由表层冰盖阻隔了大部分大气环境变化的影 
响 ${ }^{[21]}$, 冰体在相对稳定的环境中生长, 只受到光照、气温及水温变化的影响, 如果生长缓慢, 冰晶完整发育, 气泡及污染物浓度较低. 基底层冰晶延续中层, 主要以柱状冰为主, 但此时冰盖已有一定厚度, 具有隔热作 用, 加之液态水浓度增大, 冰点下降, 冰盖生长较慢, 污染物浓度显著下降. 底层是冰盖与液态水的接触面, 冰盖结构相对松散,所含污染物浓度较高.

\section{1 冰盖密度与气泡分布特征分析}

冰盖气泡与密度分布是冰体结构最直观的 2 个参数. 空气密度远小于纯冰, 所以天然冰的密度与气泡 含量呈反比. 如图 5(a) 所示, I12 冰芯纵向剖面气泡分布与垂向量密度变化具有相反的趋势. 然而二者并不 是显著的线性负相关关系, 这是由于气泡的产生受初始气体溶解量、水温、微生物作用、液态水流流速及底 栖生物产气作用等多种因素的影响. 在这些因素共同作用下, 冰盖生长中夹带的气泡呈现随着冰厚的增大 而增多的趋势. 在近底层区域出现大气泡的层状分布, 间隔为 $1.5 \sim 2.5 \mathrm{~cm}$, 这是昼夜气温与光照交替变化共 同作用的结果.

\section{2 冰盖结构和冰中污染物分布特征分析}

污染物在冰体中的迁移不仅受冰生长速率的影响, 还会受到冰体内部物理、化学和生物过程的影响. 不 同深度上气泡含量不仅影响冰盖垂向密度变化, 气泡的成因、光学作用和与晶体共存方式也使得污染物分 布与密度变化呈现出紧密的相关性.

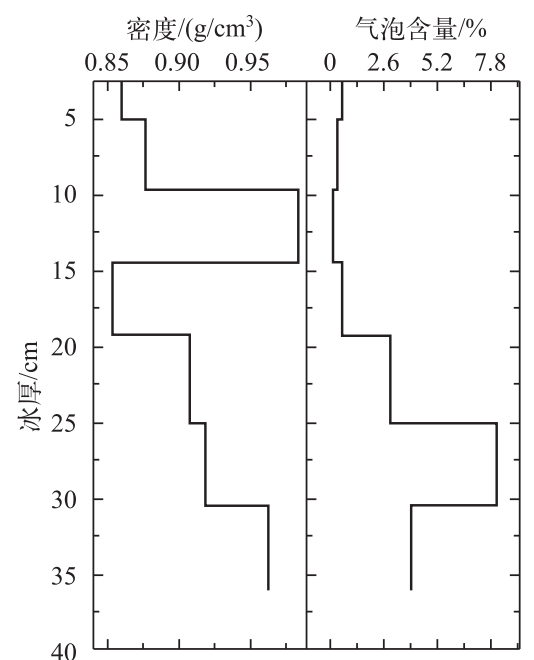

(a)

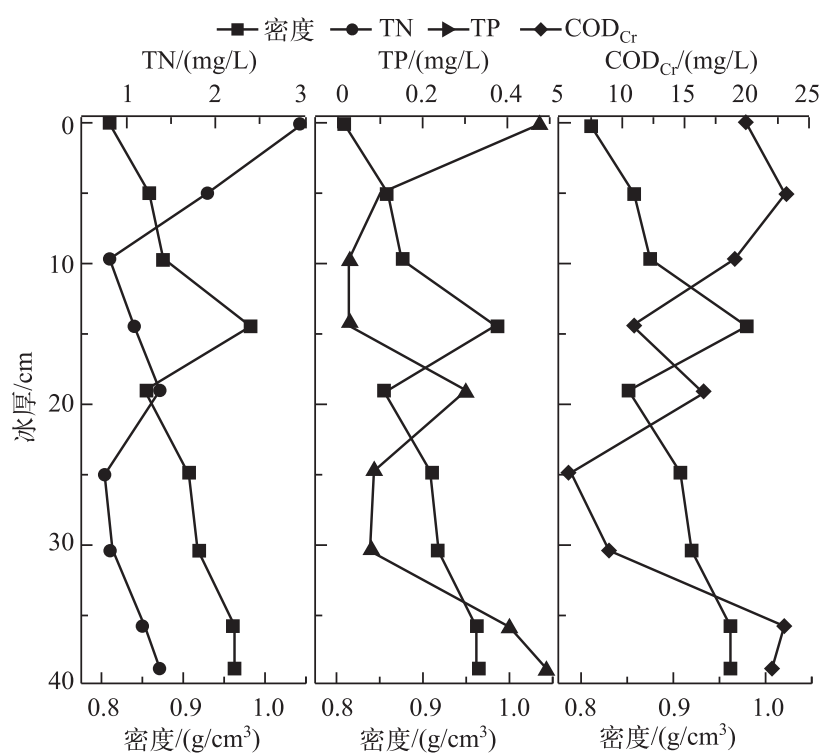

(b)

图 5 冰盖密度与气泡含量垂向分布 (a) 及密度与各污染物浓度垂向分布趋势对比(b)

Fig.5 Density and bubble percentage distribution(a) and each pollutant concentration distribution compared with density changes along vertical direction in ice core

从 $\mathrm{I} 12$ 点的 TN、TP 及 $\mathrm{COD}_{\mathrm{Cr}}$ 在冰盖中的垂向浓度分布可以看出 (图 5), 各污染物浓度与密度变化呈现 负相关关系, 对污染物浓度和冰体密度及气泡含量分别做回归分析, 发现污染物浓度与冰盖密度呈二次函 数关系, 与气泡含量呈一次函数关系, 污染物浓度分布受两者共同影响, 二者不独立, 所以建立方程形式 如下:

$$
C=a \rho^{2}+b \rho+c \rho V_{\mathrm{a}}+d V_{\mathrm{a}}+e
$$

式中, $C$ 为污染物浓度 $(\mathrm{mg} / \mathrm{L}), \rho$ 为冰体密度 $\left(\mathrm{g} / \mathrm{cm}^{3}\right) ; V_{\mathrm{a}}$ 为冰体气泡含量 $(\%) ; a 、 b 、 c 、 d 、 e$ 为拟合系数.

用 2 根较长冰芯 $\mathrm{I} 12$ (9 层) 和 $\mathrm{J} 11$ (11 层) 的冰结构数据与 $\mathrm{TN} 、 \mathrm{TP}$ 和 $\mathrm{COD}_{\mathrm{Cr}}$ 进行二元回归, 得到回归方 程, 相关系数分别为 $0.8965 、 0.8718$ 和 0.8184 (图 6). 冰盖在形成过程中, 将冰层污染物排人湖泊水体, 造成 冰下水体污染物浓缩, 利用拟合方程结合冰厚及冰盖结构数据可以对冰体排除污染物总量及冰盖对冰下水 
(a)

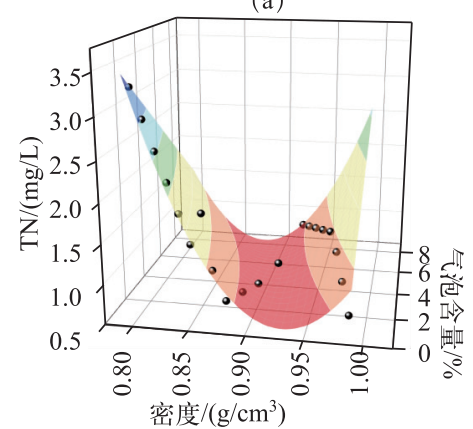

(b)

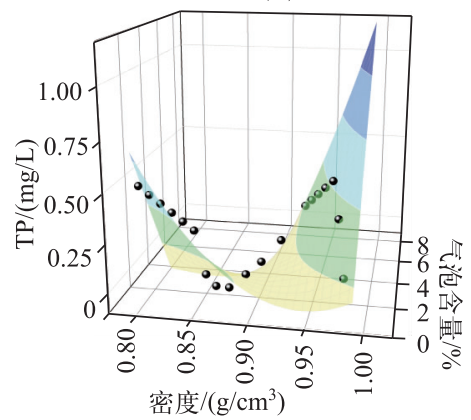

(c)

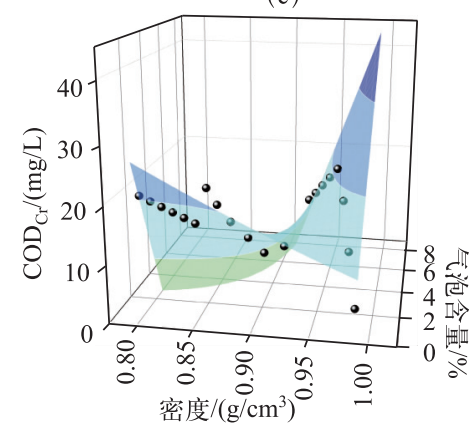

$C_{\mathrm{TN}}=168.35 \rho^{2}-313.85 \rho-2.11 \rho V_{\mathrm{a}}+C_{\mathrm{TP}}=35.91 \rho^{2}-67.97 \rho-2.09 \rho V_{\mathrm{a}}+C_{\mathrm{COD}_{\mathrm{Cr}}}=149.06 \rho^{2}-375.82 \rho-83.51 \rho V_{\mathrm{a}}+$
$2.33 V_{\mathrm{a}}+146.87, r_{\mathrm{TN}}=0.8965$
$2.30 V_{\mathrm{a}}+32.13, r_{\mathrm{TP}}=0.8718$
$89.83 V_{\mathrm{a}}+234.82, r_{\mathrm{COD}_{\mathrm{Cr}}}=0.8184$

图 6 冰结构特征与污染物垂向变化关系拟合图及拟合方程

Fig. 6 Surface fitting for the relation between ice structure characteristics and the pollutant vertical distributions and their fitting equation

体污染物浓缩作用进行更精确地量化分析.

\section{4 结论}

通过对 2013-2014 年湖泊冰盖冰芯物理结构及污染物浓度分布进行研究, 以蒙新高原区的乌梁素海为 研究对象, 分析冰封期湖泊冰盖物理结构特征,从物理特征的角度阐述冰盖中污染物分布规律,为今后寒区 湖泊污染物运移过程研究提供新思路.

冰盖污染物浓度垂向分布与冰体结构特征具有较好的相关关系, 结合人湖水量、水质、冰厚及建立的多 元回归模型, 可以预测冰盖在生长过程中冰下水环境的水质状况, 为冬季湖泊水环境监测及水资源调控方 案制定提供了一定的理论依据.

与其他地区的湖泊相比, 季节性冰盖对于高纬度地区湖泊的生态过程具有特殊的影响作用 ${ }^{[22]}$. 冬季冰 盖浮于液态水之上, 减少湖水热能的散失, 却也影响光照、阻隔大气与冰下水环境气体交换, 未来深人研究 湖泊冰盖的光学特征, 热通量等将有助于进一步阐明冰盖对于湖泊生态过程的影响.

致谢: 感谢“河、湖、湿地水环境保护与修复技术研究团队”的各位老师和同学在野外采样工作及论文完成过 程中所给予的无私帮助.

\section{5 参考文献}

[ 1 ] 中国科学院南京地理与湖泊研究所. 中国湖泊概论. 北京: 科学出版社, 1989:1-3.

[ 2 ] Leppäranta M. Thermodynamics of seasonal lake ice. In: Leppäranta M ed. Freezing of lakes and the evolution of their ice cover. Berlin : Springer-Heidelberg, 2015 : 91-135.

[ 3 ] Leppäranta M, Tikkanen M, Virkanen J. Observations of ice impurities in some Finnish lakes. Proceedings of the Estonian Academy of Science Chemistry, 2003, 52(2) : 59-75.

[ 4 ] Kansanen PH, Aho J. Changes in the macrozoobenthos associations of polluted Lake Vanajavesi, Southern Finland, over a period of 50 years. Annales Zoologici Fennici,1981, 18(2): 73-101.

[ 5 ] 姜慧琴. 乌梁素海营养盐在冰体中的空间分布及其在冻融过程中释放规律的试验研究 [学位论文]. 呼和浩特: 内 蒙古农业大学,2011.

[ 6 ] 王 爽. 乌梁素海冰封期重金属元素分布规律及其室内模拟试验研究 [ 学位论文]. 呼和浩特: 内蒙古农业大 学, 2012 .

[ 7 ] 张 岩, 李畅游, Shen Hungto 等. 乌梁素海湖泊冰生长过程中总氮的迁移规律. 水科学进展, 2013, (5) : 728-735.

[ 8 ] Gow AJ, Govoni JW. Ice growth on Post Pond, 1973-1982. Cold Regions Research and Engineering Laboratory Hanover 
NH, 1983.

[ 9 ] Li Zhijun, Peng Wanwei, Zhang Tao et al. Effect of porosity on the uniaxial compressive strength of ice in Liaodong Gulf. 10th International Symposium on Okhotsk Sea, Sea Ice \& People. Hokkaido, 1995: 358-363.

[10] Huang W, Li Z, Han H et al. Structural analysis of thermokarst lake ice in Beiluhe Basin, Qinghai-Tibet Plateau. Cold Regions Science and Technology, 2012, 72: 33-42.

[11] Shi L, Li Z, Feng E et al. Thermal diffusivity identification of distributed parameter systems to sea ice. Journal of Applied Mathematics, 2013, special issue: 499-520.

[12] 李志军, 贾 青, 黄文峰等. 水库淡水冰的晶体和气泡及密度特征分析. 水利学报, 2009, 11: 1333-1338.

[13] 国家环境保护总局《水和废水监测分析方法》编委会. 水和废水监测分析方法: 第 4 版. 北京: 中国环境科学出版 社, 2002.

[14] Gow AJ. Orientation textures in ice sheets of quietly frozen lakes. Journal of Crystal Growth, 1986, 74(2) : 247-258.

[15] Salonen K, Leppäranta M, Viljanen M et al. Perspectives in winter limnology : closing the annual cycle of freezing lakes. Aquatic Ecology, 2009, 43(3): 609-616.

[16] Perovich DK, Roesler CS, Pegau WS. Variability in Arctic sea ice optical properties. Journal of Geophysical Research: Oceans $(1978-2012), 1998,103$ ( C1) : 1193-1208.

[17] 滕 晖, 邓 云, 黄奉斌等. 水库静水结冰过程及冰盖热力变化的模拟试验研究. 水科学进展, 2011, 22(5): $720-726$.

[18] Leppäranta M. Landice interaction in the Baltic Sea. Estonian Journal of Earth Sciences, 2013, 62(1) : 2-14.

[19] Zhang G, Sun A. Study on the growth mechanism and microstructure of a heavy hail in the eastern part of Qinghai Province. Plateau Meteorology, 2007, 4: 15.

[20] Usowicz B, Lipiec J, Usowicz JB. Thermal conductivity in relation to porosity and hardness of terrestrial porous media. Planetary and Space Science, 2008, 56(3): 438-447.

[21] Arst H, Erm A, Herlevi A et al. Optical properties of boreal lake waters in Finland and Estonia. Boreal Environment Research, 2008, 13(2): 133-158.

[22] Salonen K, Leppäranta M, Viljanen M et al. Perspectives in winter limnology : closing the annual cycle of freezing lakes. Aquatic Ecology, 2009, 43(3):609-616. 